\title{
Consistencia interna y dimensionalidad del cuestionario breve para detectar intimidación escolar ${ }^{1}$
}

\author{
Guillermo A. Ceballos-Ospino², Adalberto Campo-Arias ${ }^{3}$ y Ubaldo Rodríguez-De Ávila ${ }^{4}$ \\ Universidad del Magdalena (Colombia)
}

Recibido: 13/07/2019Ａceptado: 23/06/2020

\begin{abstract}
Resumen
Objetivo. Corroborar el desempeño psicométrico, la consistencia interna y la dimensionalidad del Cuestionario Breve de Detección de Intimidación Escolar (CBDIE). Método. Se diseñó un estudio de validación en el que participaron 350 estudiantes, entre los 10 y los 17 años $(M=13.3 ; D E=1.8), 53.7 \%$ de género femenino. Se calcularon indicadores de consistencia interna (Kuder-Richardson, K-R, y omega de McDonald) para las tres subescalas del CBDIE. Resultados. La subescala de intimidación percibida mostró K-R de 0.55 y omega de McDonald de 0.58; la subescala de síntomas emocionales, 0.80 y 0.81 ; y la subescala de intimidación perpetrada, 0.41 y 0.33 . No fue posible demostrar la dimensionalidad del instrumento. Conclusión. Solo la subescala de síntomas emocionales de CBDIE presenta alta consistencia interna. Se necesitan más investigaciones de la dimensionalidad del CBDIE, para dar cuenta de su estructura interna.
\end{abstract}

Palabras clave. Intimidación escolar, validez y confiabilidad, adolescentes, estudiantes, estudios de validación.

\section{Internal Consistency and Dimensionality in the Brief Questionnaire for Bullying Detection}

\begin{abstract}
Objective. To corroborate the psychometric performance, internal consistency and dimensionality, of the Brief Questionnaire for Bullying Detection (BQBD). Method. A validation study was designed in which 350 students between 10 and 17 years of age $(M=13.3 ; S D=1.8)$ participated, $53.7 \%$ of them female. Internal consistency indicators (Kuder-Richardson, K-R and McDonald's omega) were calculated for the three sub-scales of the BQBD. Results. The sub-scale of perceived intimidation showed K-R of 0.55 and McDonald's omega of 0.58; the sub-scale of emotional symptoms were 0.80 and 0.81 , and the sub-scale of perpetrated intimidation were 0.41 and 0.33 , respectively. It was impossible to demonstrate the proposed dimensionality. Conclusion. Only the sub-scale of emotional symptoms of the BQBD has high internal consistency. More research on the dimensionality of the BQBD is needed to explain its internal structure.

Keywords. Bullying, validity and reliability, adolescent, students, validation studies.

Este proyecto fue aprobado y financiado por la Universidad del Magdalena, Santa Marta, Colombia.

2 Psicólogo especialista en Gerencia de la calidad en servicios de salud. Programa de Psicología de la Universidad del Magdalena. Correo de correspondencia: guillermoceballos@gmail.com / gceballos@unimagdalena.edu.co

3 Magíster en Salud Sexual y Repdroductiva.

4 Doctor en Psicobiología
\end{abstract}




\title{
Consistência interna e dimensionalidade do questionário breve para a detecção da intimidação escolar
}

\begin{abstract}
Resumo
Escopo. Corroborar o desempenho psicométrico, a consistência interna e a dimensionalidade do Questionário Breve de Detecção de Intimidação Escolar (CBDIE). Metodologia. Foi desenhado um estudo de validação no que participaram 350 estudantes, entre os 10 e os 17 anos $(M=13.3 ; D E=1.8) ; 53.7 \%$ de gênero feminino. Foram calculados indicadores de consistência interna (Kuder-Richardson, K-R, e ômega de McDonald) para as três sub-escadas do CBDIE. Resultados. A sub-escada de intimidação percebida mostrou K-R de 0.55 e ômega de McDonald de 0.58; a sub-escada de sintomas emocionais, 0.8 e 0.81 ; e a sub-escada de intimidação perpetrada, 0.41 e 0.33. Não foi possível demostrar a dimensionalidade do instrumento. Conclusão. Só a sub-escada de sintomas emocionais de CIBDE apresenta alta consistência interna. Foram necessitadas mais pesquisas da dimensionalidade do CIBDE, para dar conta da sua estrutura interna.
\end{abstract}

Palavras-chave. Intimidação escolar, validade e confiabilidade, adolescentes, estudantes, estudos de validação.

\section{Introducción}

El desempeño de los instrumentos de medición en salud presenta variaciones en los indicadores psicométricos, según las características de la población (Keszei, Novak y Streiner, 2010). Por lo tanto, es necesario observar, revisary corroborar estos indicadores en distintas poblaciones en diferentes oportunidades para garantizar la estabilidad y la validez del constructo evaluado (Alarcón y Muñoz, 2008; Simancas-Pallares, Herazo y Campo-Arias, 2016). Con frecuencia, algunos instrumentos de medición solo muestran un rendimiento excelente en manos de los investigadores que los diseñaron y no durante las aplicaciones posteriores en estudios en otros contextos. Estos hallazgos se presentan por diferentes motivos: las características de la población, la traducción a otro idioma, o las adaptaciones o modificaciones culturales realizadas. Por esta razón, es fundamental examinar repetidamente el rendimiento en diferentes contextos y grupos poblacionales (Guimaraes, Haas, Spadoti, Marques dos Santos y Galvão, 2017; Muñiz, Elosua y Hambleton, 2013; Ramada-Rodilla, Serra-Pujadas y Delclós-Clanchet, 2013).

En cuanto a las distintas formas de violencia en el contexto escolar, los instrumentos de medición en salud y social son herramientas que permiten estudiar el fenómeno, que si bien ha estado presente anteriormente, solo logró atención en las últimas décadas (Serrano e Iborra, 2005), debido a que la denuncia de estos hechos se ha incrementado notablemente (Analitis et al., 2009; CassianiMiranda, Gómez-Alhach, Cubides-Munévar y Hernández-Carrillo, 2014; Cepeda, Pacheco, García y Piraquive, 2008; Cuevas, Hoyos y Ortiz, 2009; Kim y Chun, 2020; Modecki, Minchin, Harbaugh, Guerra y Runions, 2014; Walters, 2020). Hoy se considera la intimidación o matoneo escolar como un problema de salud pública, dadas las consecuencias negativas en el bienestar general del estudiantado, en morbilidad y mortalidad, vandalismo, comportamientos disociales, escaparse y abandonar la escuela, consumo de sustancias legales e ilegales, síntomas depresivos e incluso ideación suicida (Analitis et al., 2009; Chaux, 2003; Hawker y Boulton, 2000; Heikkilä et al., 2013; Kim y Chun, 2020; Ortega y Monks, 2005; Srabstein, Berkman y Pyntikova, 2008). La intimidación escolar se puede presentar de dos formas: (a) maltrato físico, verbal o exclusión social en forma repetida, sistemática; y (b) cuando es evidente una clara diferencia de estatus de poder entre agresor y víctima (Collell y Escudé, 2002).

El término intimidación escolar difiere según el país en que se estudie el acontecimiento: en España, luego de un consenso entre los investigadores del tema, se le llama bullying (Defensor del Pueblo, 2007), igual que en países de habla inglesa como Estados Unidos (Green, 2007). En Latinoamérica se le conoce de formas diferentes (Collell y Escudé, 2003; Pautasso, 2006); por ejemplo, en Colombia se le llama intimidación, acoso, abuso o violencia escolar (Chaux, 2003; Paredes, Álvarez, Lega y 
Vernon, 2008) y en Argentina, patoterismo (Torres, 2005).

La agresión, según Bandura (1973), es una respuesta a un estímulo provocador, un comportamiento perjudicial y destructivo. Mientras que Berkowitz (1996) la concibió como un comportamiento con intención de herir física y psicológicamente a otro, a sabiendas de que la acción no es agradable para la persona a quien se dirige, lo cual le da connotaciones de intencionalidad. Por su parte, Camargo (1996) describe el tipo de violencia escolar como aquel que realizan estudiantes contra sus pares $y$, en ocasiones, va dirigida contra maestros en forma de amenazas, lesiones, actitudes desafiantes y hasta homicidios.

Los diversos tipos de intimidación van muy acordes al género, ya que la frecuencia suele ser un poco mayor entre los hombres (Card, Stucky, Sawalani y Little, 2008), quienes tienden a utilizar el maltrato físico y directo; mientras que las mujeres hacen más uso de la intimidación psicológica y verbal y prefieren la agresividad indirecta (Card et al., 2008; Cerezo, 2001; Oñate-Cantero y Piñuel, 2005; Ortega y Monks, 2005; Serrano e Iborra, 2005).

Dadas las características del fenómeno, se han diseñado varios cuestionarios o escalas para cuantificar en forma válida y confiable sus diversas aristas desde distintas aproximaciones teóricas, lo que puede influenciar el desempeño psicométrico. Entre otros se tienen: el Instrumento de Relaciones entre Pares Adolescentes, la Escala de Agresión, la Escala de California de Victimización e Intimidación y el Cuestionario de Olweus de Intimidación (VivoloKantor, Martell, Holland y Westby, 2014). Para el contexto colombiano, Moratto, Cárdenas y Berbesí (2012) diseñaron e informaron el desempeño psicométrico del Cuestionario Breve para Detectar Intimidación Escolar (CBDIE) en una muestra no probabilística de 512 estudiantes de Medellín, en edades entre 11 y 18 años. Este instrumento se compone de 36 ítems que fueron retenidos de una escala de mayor extensión, mediante análisis factorial exploratorio (AFE), empleando el método de componentes principales. Los ítems se distribuyeron en tres dimensiones o subescalas de 12 ítems cada una: (a) intimidación percibida, (b) síntomas emocionales (ansiosos, depresivos, estrés postraumático y baja autoestima), e (c) intimidación perpetrada que mostró una consistencia interna de $0.87,0.89$ y 0.83 , respectivamente, con alfa de Cronbach.

El análisis anterior tiene cuatro limitaciones: la primera, que la extracción de los ítems se realizó por el método de componentes principales, el cual está indicado para la reducción del número de un conjunto de variables y no para establecer un rasgo latente en un grupo de ítems como los que exploran las escalas de medición de constructos (Jackson, Gillaspy y Purc-Stephenson, 2009; Streiner, 2003). La segunda, no se consideró la limitación del análisis factorial para la interpretación de soluciones factoriales para paquete de ítems superior a 30, generalmente; en estos casos, es poco probable que se reproduzcan las dimensiones teóricas pensadas (Campo-Arias, Herazo y Oviedo, 2012; Streiner, 1994). La tercera, los autores omitieron la presentación de un análisis factorial confirmatorio (AFC) para cada una de las dimensiones, con el fin de conocer si los datos analizados se ajustaban al modelo propuesto de las tres dimensiones antes anotadas (Brown, 2006; Reise, Waller y Comrey, 2000). Por último, se omitió el cálculo de un segundo estimador de consistencia interna, como omega de McDonald, que brinda un coeficiente más preciso de la relación entre los ítems, cuando se viola el principio de tau equivalencia. Es decir, cuando ellos muestran distintos valores de coeficientes que se corroboran en grandes diferencias en la solución factorial (Campo-Arias y Oviedo, 2008; Hogan, Benjamin y Brezinski, 2000).

Dadas las falencias mencionadas, fue necesario probar el rendimiento psicométrico del CBDIE en otra muestra de estudiantes colombianos, con técnicas más adecuadas para la naturaleza compleja del constructo intimidación escolar. Esta observación se requiere para garantizar la utilidad práctica del instrumento y la utilización del cuestionario en futuras investigaciones en grupos poblacionales similares del Caribe colombiano que exploren las tres dimensiones planteadas teóricamente para intimidación escolar en el CBDIE (Sánchez y Echeverry, 2004). En ese sentido, el objetivo de este estudio fue corroborar el desempeño psicométrico, la consistencia interna y la dimensionalidad del CBDIE en estudiantes adolescentes en Santa Marta, departamento del Magdalena, Colombia. 


\section{Método}

\section{Diseño}

Se diseñó un estudio de validación que contó con la aprobación de un comité de ética en investigación (aprobación firmada el 18 de junio de 2017).

\section{Participantes}

Se tomó una muestra no probabilística por conveniencia de 350 estudiantes de dos colegios de Santa Marta, Magdalena (Colombia), uno público y otro privado. La edad estaba entre 10 y 17 años $(M=13.3, D E=1.8)$. Según el género, la muestra se distribuyó en 188 participantes (53.7\%) de género femenino y $162(46.3 \%)$ del masculino. Respecto al nivel de escolaridad, 236 estudiantes (67.4\%) de básica secundaria (sexto a noveno grado) y 114 (32.6\%) de media vocacional (décimo y undécimo grado).

\section{Instrumento}

Los participantes completaron el CBDIE el cual estaba compuesto por tres subescalas que exploran intimidación percibida, síntomas emocionales (ansiedad, depresión, estrés postraumático y baja autoestima) e intimidación perpetrada. Cada subescala estaba conformada por 12 ítems, cada una con tres opciones de respuesta: nunca, pocas veces y muchas veces (Moratto et al., 2012). Para simplificar el patrón de respuesta, durante el último año académico se optó por un patrón de respuesta más simple, dicotómico: sí o no. A cada respuesta afirmativa se le calificó con uno (1) y la respuesta negativa con cero (0). Estos patrones de respuesta no modifican significativamente el desempeño de la mayoría de instrumentos de medición (Domínguez, 2013; López, 2005).

\section{Análisis de datos}

\section{Consistencia interna.}

La consistencia interna es una medida directa de confiabilidad e indirecta de validez, que indica la correlación media entre los ítems que componen una escala. Para escalas multidimensionales, se recomienda que se calcule en forma separada para cada subescala. Los valores de consistencia interna se ubican entre 0 y 1.0, y según el desarrollo y la experiencia con el instrumento, se aceptan valores entre 0.70 y 0.95 (Campo-Arias y Oviedo, 2008; Keszei et al., 2010).

Se calculó la consistencia interna para cada subescala con la prueba o fórmula 40 de KuderRichardson (K-R) para patrones de respuesta dicotómica (Kuder y Richardson, 1937). Esta prueba es exactamente equivalente al coeficiente de alfa de Cronbach (1951) que se estima para escalas con respuesta ordinal. Igualmente, se determinó el coeficiente de omega de McDonald (1970), como una segunda medida que aventaja a las pruebas antes anotadas, que subestiman la correlación entre los ítems cuando los mismos presentan coeficientes (loadings) dispares (Campo-Arias y Oviedo, 2008; Hogan et al., 2000).

\section{Dimensionalidad.}

La técnica más apropiada para demostrar la dimensionalidad de las escalas de medición es el análisis factorial. En las fases iniciales del diseño de nuevos instrumentos se indica la realización de pruebas exploratorias (AFE) que decantan los ítems para cada dimensión, y para las escalas más usadas o probadas se necesita la aproximación confirmatoria (AFC) para garantizar la dimensionalidad o el refinamiento de los ítems que mejor recogen el aspecto esencial de cada dimensión (Brown, 2006; Campo-Arias et al., 2012).

Se realizó el AFE como una aproximación a la dimensionalidad de cada subescala del CBDIE, se calcularon los coeficientes de KMO (Kaiser, 1974), prueba de Bartlett (1950), valor propio para el factor y varianza explicada. Para ello, se eligió el método de máxima verosimilitud indicado para el estudio de un conjunto de ítems a los que subyace un factor o rasgo latente. En el AFC se estimaron indicadores de bondad de ajuste que aceptan o rechazan la dimensionalidad observada en el AFE. Se calcularon chi cuadrado, RMSEA (raíz del cuadrado medio del error de aproximación), CFI (índice comparativo de ajuste) y TLI (índice de Tucker-Lewis). El valor de chi cuadrado es adecuado si el valor de probabilidad (p) es superior al 5\%; RMSEA es aceptable si es menor de 0.06, y CFI y TLI se esperan con valores superiores a 0.89. El análisis se realizó en Stata 13.0 (2013). 


\section{Consideraciones éticas.}

Los padres de familia firmaron el consentimiento informado y los estudiantes dieron el asentimiento para formar parte de la investigación, en consonancia con la Resolución 8430 que reglamentó la investigación en salud (Ministerio de Salud de Colombia, 1993).

\section{Resultados}

\section{Consistencia interna}

La subescala de intimidación percibida mostró coeficiente K-R de 0.55 y omega de McDonald de 0.58; para la subescala de síntomas emocionales,
K-R fue de 0.80 y omega de McDonald de 0.81; y la subescala de intimidación perpetrada mostró K-R de 0.41 y omega de McDonald de 0.33 .

\section{Dimensionalidad}

En el AFE, el KMO para la subescala de intimidación percibida fue de 0.60 , para síntomas emocionales 0.87 , y para intimidación perpetrada no se pudo calcular, dado que para algunos ítems la varianza fue cero. El valor de chi cuadrado para intimidación percibida fue 579.6, para síntomas emocionales, 909.9, y para intimidación perpetrada (en ambos casos, $g l=66$ y $p<0.01$ ), no se pudo calcular, pues no se alcanzó convergencia. Los indicadores de bondad de ajuste del AFC se presentan en la tabla 1.

Tabla 1

Indicadores de la bondad de ajuste del modelo unidimensional de las subescalas

\begin{tabular}{lcccc}
\hline Subescala & Chi cuadrado $(g l, p)^{*}$ & RMSEA (IC90\%) & CFI & TLI \\
\hline Intimidación percibida & $391.6(2,0.01)$ & $0.12(0.11-0.13)$ & 0.47 & 0.35 \\
Síntomas emocionales & $146.1(64,0.01)$ & $0.07(0.06-0.08)$ & 0.89 & 0.87 \\
Intimidación perpetrada & $\mathrm{Cl}$ & $\mathrm{Cl}$ & $\mathrm{Cl}$ & $\mathrm{Cl}$ \\
\hline
\end{tabular}

Nota. Cl: cálculo imposible. Fuente: elaboración propia.

\section{Discusión}

Con el fin de corroborar el desempeño psicométrico, la consistencia interna y la dimensionalidad del Cuestionario Breve de Detección de Intimidación Escolar (CBDIE), el análisis que se llevó a cabo puso en evidencia un desempeño global pobre para las subescalas del CBDIE. Asimismo, los coeficientes de consistencia interna fueron bajos, excepto para la subescala de síntomas emocionales, y en el AFC no se logró corroborar la dimensionalidad de las subescalas.

Los resultados mostraron que el rendimiento psicométrico de las escalas de medición es altamente variable según el contexto y las características sociodemográficas de la población participante. También se encontró una consistencia interna entre 0.41 y 0.81 para las subescalas del CBDIE. Las subescalas de intimidación perpetrada y percibida evidenciaron una limitada consistencia interna, tanto por K-R como por omega de McDonald. Estos hallazgos difieren radicalmente de las observaciones de Moratto et al. (2012), quienes informaron valores de alfa de Cronbach entre 0.83 y 0.89 .

Esta disparidad entre los estudios no es poco frecuente en estudios metodológicos, puesto que se entiende que el patrón de respuesta es directamente dependiente de las características de la población y no necesariamente una propiedad intrínseca de los instrumentos (Blais y Baer, 2010; CampoArias y Oviedo, 2008; Streiner, 2003). Los valores de consistencia interna se suelen aceptar como adecuados, si se encuentran en el rango de 0.70 a 0.95, según el grado de desarrollo y el tipo de constructo que pretende medir el instrumento (Keszei et al., 2010; Oviedo y Campo-Arias, 2005).

La dimensionalidad de las escalas de medición guarda una relación directa con los valores en los coeficientes de consistencia interna. En el presente estudio, la dimensionalidad no satisfizo los parámetros concertados; además, no existen análisis precedentes que permitan una comparación 
técnicamente aceptable. No obstante, el análisis de factores es la técnica estadística más usada para probar la dimensionalidad de instrumentos como el CBDIE, que se acepta también como una forma de validez de constructo (Streiner, 1994). Esto implica que la no confirmación de la dimensionalidad invita a la revisión de la teoría que soporta el instrumento o el examen de los ítems que lo componen (CampoArias et al., 2012; Reise et al., 2000). La validación de las escalas de medición es un proceso permanente de revisiones y adaptaciones, según los hallazgos en las distintas muestras estudiadas (Jackson et al., 2009; Streiner, 2003).

Las diferencias observadas entre el presente estudio y el precedente de Moratto et al. (2012) se deben a los distintos patrones de respuesta usados en cada estudio, dicotómico frente a politómico. Sin embargo, esta teoría queda sin fundamento dada la alta consistencia que presentó la subescala de síntomas emocionales. Adicionalmente, datos empíricos documentaron que el número posible de opciones de respuesta afecta poco a estos coeficientes (Domínguez, 2013; López, 2005).

Igualmente, los hallazgos confirman la necesidad de conocer el desempeño psicométrico de un instrumento en una población específica. Es decir, no se puede asumir o confiar en validaciones realizadas en otras poblaciones, pues en los estudios metodológicos es imposible la generalización, aunque la muestra sea probabilística (Campo-Arias y Oviedo, 2008; Keszei et al., 2010; Streiner, 2003).

En esta investigación se realizó una evaluación psicométrica más exhaustiva, lo cual supone una ventaja. Se calculó un segundo coeficiente de consistencia interna y se llevó a cabo AFC que evidencia la necesidad de las evaluaciones psicométricas periódicas (Reise et al., 2000). No obstante, es necesario corroborar estos hallazgos en otras investigaciones.

En conclusión, las subescalas del CBDIE presentaron diferencias marcadas en la consistencia interna. Únicamente la subescala de síntomas emocionales mostró alta consistencia interna. Las soluciones unidimensionales de las subescalas no se ajustaron a los datos en esta muestra de estudiantes de Santa Marta, Colombia. En consecuencia, no se recomienda la aplicación para la medición del constructo. Se necesitan nuevas investigaciones que revisen, redefinan o refinen las subescalas actuales.

\section{Referencias}

Alarcón, A. M. y Muñoz, S. (2008). Medición en salud: Algunas consideraciones metodológicas. Revista Médica de Chile, 136, 125-130. doi:10.4067/S0034-98872008000100016

Analitis, F., Velderman, M. K., Ravens-Sieberer, U., Detmar, S., Erhart, M., Herdman, M.,... Rajmil, L. (2009). Being Bullied: Associated Factors in Children and Adolescents 8 to 18 Years Old in 11 European Countries. Pediatrics, 123, 569577. doi:10.1542/peds.2008-0323

Bandura, A. (1973). Aggression: A Social Learning Analysis. Englewood Cliffs: Prentice Hall.

Bartlett, M. S. (1950). Test of Significance in Factor Analysis. British Journal of Psychology, 3, 7785. doi:10.1111/j.2044-8317.1950.tb00285.x

Berkowitz, L. (1996). Agresión: Causas, consecuencias y control. Bilbao: Desclee de Brouwer.

Blais, M., \& Baer, L. (2010). Understanding Rating Scales and Assessment Instruments. In M. Blais, \& L. Baer, Handbook of Clinical Rating Scales and Assessment in Psychiatry and Mental Health (pp. 1-7). New York: Humana Press.

Brown, T. A. (2006). Confirmatory factor analysis for applied research. New York: The Guilford Press.

Card, N. A., Stucky, B. D., Sawalani, G. M., \& Little, T. D. (2008). Direct and Indirect Aggression during Childhood and Adolescence: A Meta Analytic Review of Gender Differences, Intercorrelations, and Relations to Maladjustment. Child Development, 79(5), 1185-1229. doi:10.1111/ j.1467-8624.2008.01184.x

Camargo, A. M. (1996). Violencia escolar y violencia social. V Congreso Nacional de Prevención y Atención del Maltrato Infantil. Red Académica, Universidad Nacional, Bogotá.

Campo-Arias, A., Herazo, E. y Oviedo, H. C. (2012). Análisis de factores: fundamentos para la evaluación de instrumentos de medición en salud mental. Revista Colombiana de Psiquiatría, 41, 659-671.

Campo-Arias, A. y Oviedo, H. C. (2008). Propiedades psicométricas de una escala: la consistencia interna. Revista de Salud Pública, 10(5), 831-839. 
Cassiani-Miranda, C. A., Gómez-Alhach, J., Cubides-Munévar, A. M. y Hernández-Carrillo, M. (2014). Prevalencia de bullying y factores relacionados en estudiantes de bachillerato de una institución educativa de Cali, Colombia, 2011. Revista de Salud Pública, 16(1), 14-26. doi:10.15446/rsap.v16n1.43490

Cepeda, E., Pacheco, P. N., García, L. y Piraquive, C. L. (2008). Acoso escolar a estudiantes de educación básica y media. Revista de Salud Pública, 10(4), 517-528.

Cerezo, F. (2001). Variables de personalidad asociadas en la dinámica bullying (agresores versus víctimas) en niños y niñas de 10 a 15 años. Revista Anales de Psicología, 17(1), 3743.

Chaux, E. (2003). Agresión reactiva, agresión instrumental, el ciclo de la violencia. Revista de Estudios Sociales, 15, 47-58. doi:10.7440/ res15.2003.03

Collell, J. y Escudé, C. (2002). La violencia entre iguals a l'escola: el bullying. Ámbits de Psicopedagogía, 4, 20-24.

Collell, J. y Escudé, C. (2003). L'educación emocional. Revista dels Mestres de la Garrotxa, 37, 8-12.

Colombia. Ministerio de Salud. (1993). Resolución 008430. Por la cual se establecen las normas científicas, técnicas y administrativas para la investigación en salud. Bogotá, Colombia.

Cronbach, J. (1951). Coefficient Alpha and the Internal Structure of Test. Psychometrika, 16(3), 297-334.

Cuevas, M. C., Hoyos, P. A. y Ortiz, Y. (2009). Prevalencia de intimidación en dos instituciones educativas del departamento del Valle del Cauca. Pensamiento Psicológico, 6(13), 153-172.

Defensor del Pueblo. (2007). Informes, estudios y documentos: Violencia escolar: El maltrato entre iguales en la Educación Secundaria Obligatoria 1999-2006 (Nuevo estudio y actualización del informe 2000). Madrid: Autor.

Domínguez, S. A. (2013). ¿ĺtems politómicos o dicotómicos? Un estudio empírico con una escala unidimensional. Revista Argentina de Ciencias del Comportamiento, 5(3), 30-37.
Green, G. (2007). Bullying: A Concern for Survival. Education, 128(2), 333-337.

Guimaraes, M. B., Haas, V. J., Spadoti, R. A., Marques dos Santos, M., \& Galvão, C. M. (2017). Cultural Adaptation and Validation of an Instrument on Barriers for the Use of Research Results. Revista Latino-Americana da Enfermagem, 25, e.2852. doi:10.1590/15188345.1652 .2852

Hawker, D. S., \& Boulton, M. J. (2000). Twenty Years' Research on Peer Victimization and Psychosocial Maladjustment: A Meta-Analytic Review of Cross-Sectional Studies. The Journal of Child Psychology and Psychiatry and Allied Disciplines, 41(4), 441-455. doi:10.1111/14697610.00629

Heikkilä, H-K., Väänänen, J., Helminen, M., Fröjd. S., Marttunen, M., \& Kaltiala-Heino, R. (2013). Involvement in bullying and suicidal ideation in middle adolescence: a 2-year follow-up study. European Child \& Adolescent Psychiatry, 22(2), 95-102. doi: 10.1007/s00787-012-0327-0

Hogan, T. P., Benjamin, A., \& Brezinski, K. L. (2000). Reliability Methods: A Note on the Frequency of Use of Various Types. Educational and Psychological Measurement, 60(4), 523-531. doi:10.1177/00131640021970691

Jackson, D. L., Gillaspy, J. A., \& Purc-Stephenson, R. (2009). Reporting Practices in Confirmatory Factor Analysis: An Overview and Some Recommendations. Psychological Methods, 14(1), 6-23. doi:10.1037/a0014694

Kaiser, H. F. (1974). An Index of Factorial Simplicity. Psychometrika, 34(1), 31-36. doi:10.1007/ BF02291575

Keszei, A. P., Novak, M., \& Streiner, D. L. (2010). Introduction to Health Measurement Scales. Journal of Psychosomatic Research, 68(4), 319323. doi:10.1016/j.jpsychores.2010.01.006

Kim, H. H. S., \& Chun, J. (2020). Bullying Victimization, School Environment, and Suicide Ideation and Plan: Focusing on Youth in Low and Middle Income Countries. Journal of Adolescent Health, 66(1), 115-122. doi:10.1016/j.jadohealth.2019.07.006

Kuder, G. F., \& Richardson, M. W. (1937). The Theory of the Estimation of Test Reliability. 
Psychometrika, 2, 151-160. doi:10.1007/ BF02288391

López, J. A. (2005). Ítems politómicos vs. dicotómicos: Un estudio metodológico. Anales de Psicología, 21(2), 339-344.

McDonald, R. P. (1970). Theoretical Foundations of Principal Factor Analysis and Alpha Factor Analysis. British Journal of Mathematics and Statistical Psychology, 23(1), 1-21. doi:10.1111/j.2044-8317.1970.tb00432.x

Modecki, K. L., Minchin, J., Harbaugh, A. G., Guerra, N. G., \& Runions, K. C. (2014). Bullying Prevalence across Contexts: A Meta-Analysis Measuring Cyber and Traditional Bullying. Journal of Adolescent Health, 55(5), 602-611. doi:10.1016/j.jadohealth.2014.06.007

Moratto, N. S., Cárdenas, N. y Berbesí, D. Y. (2012). Validación de un cuestionario breve para detectar intimidación escolar. CES Psicología, 5(2), 70-78.

Muñiz, J., Elosua, P. y Hambleton, R. K. (2013). Directrices para la traducción y adaptación de los tests: segunda edición. Psicothema, 25(2), 151-157. doi:10.7334/psicothema2013.24

Oñate-Cantero, A. y Piñuel, I. (2005). Informe cisneros VII. Violencia escolar en alumnos de Primaria, Eso y Bachiller, informe preliminar. España: Institución de Innovación Educativa y Desarrollo Directivo. Recuperado de https://www.bienestaryproteccioninfantil. es/imagenes/tablaContenidos03SubSec/ informe\%20cisneros\%20VII.pdf

Ortega, R. y Monks, C. (2005). Agresividad injustificada entre preescolares. Psicothema, 17(3), 453-458.

Oviedo, H. C. y Campo-Arias, A. (2005). Aproximación al uso del coeficiente alfa de Cronbach. Revista Colombiana de Psiquiatría, 34(4), 572-580.

Paredes, M. T., Álvarez, M. C., Lega, L. I. y Vernon, A. (2008). Estudio exploratorio sobre el fenómeno del bullying en la ciudad de Cali, Colombia. Revista Latinoamérica de Ciencias Sociales, Niñez y Juventud, 6(1), 295- 317.

Pautasso, N. D. (2006). La intimidación entre los niños en edad escolar. Estudio sobre las sutiles prácticas de la violencia en el ámbito de la escuela general básica. Argentina: Universidad Nacional de La Patagonia Austral.

Ramada-Rodilla, J. M., Serra-Pujadas, C. y DelclósClanchet, G. L. (2013). Adaptación cultural y validación de cuestionarios de salud: revisión y recomendaciones metodológicas. Salud Pública de México, 55(1), 57-66. doi:10.1590/ S0036-36342013000100009

Reise, S. P., Waller, N. G., \& Comrey, A. L. (2000). Factor Analysis and Scale Revision. Psychological Assessment, 12(3), 287-297. doi:10.1037/1040-3590.12.3.287

Sánchez, R. y Echeverry, J. (2004). Validación de escalas de medición en salud. Revista de Salud Pública, 6(3), 302-318.

Serrano, A. e Iborra, I. (2005). Informe Violencia entre compañeros en la escuela (Serie de documentos No. 9). Madrid: Centro Reina Sofía para el Estudio de la Violencia.

Simancas-Pallares, M. A., Herazo, E. y Campo-Arias, A. (2016). Técnicas para estimar la estabilidad de una escala de medición en salud. Revista Ciencias Biomédicas, 7(1), 104-111.

Srabstein, J. C., Berkman, B. E., \& Pyntikova, E. (2008). Antibullying Legislation: A Public Health Perspective. Journal of Adolescent Health, 42(1), 11-20. doi:10.1016/j. jadohealth.2007.10.007

StataCorp. (2013). Stata Statistical Software: Release 13. College Station, TX: StataCorp LP.

Streiner, D. L. (1994). Figuring Out Factors: The Use and Misuse of Factor Analysis. Canadian Journal of Psychiatry, 39(1), 135-140. doi:10.1177/070674379403900303

Streiner, D. L. (2003). Being Inconsistent about Consistency: When Coefficient Alpha does and doesn't Matter. Journal of Personality Assessment, 80(3), 217-222.

Torres, C. C. (2005). Jóvenes y violencia. Revista Iberoamericana de Educación, Violencia y Escuela, 37, 22-37. doi:10.35362/rie370851

Vivolo-Kantor, A. M., Martell, B. N., Holland, K. M., \& Westby, R. (2014). A Systematic Review and Content Analysis of Bullying and CyberBullying Measurement Strategies. Aggression and Violent Behavior, 19(4), 423-434. doi:10.1016/j.avb.2014.06.008 
Walters, G. D. (2020). School-Age Bullying Victimization and Perpetration: A MetaAnalysis of Prospective Studies and Research.
Trauma, Violence, \& Abuse, $X X(X), 1-11$. doi. org/10.1177/1524838020906513

Para citar este artículo / To cite this article / Para citar este artigo: Ceballos-Ospino, G. A., Campo-Arias, A. y Rodríguez-De Ávila, U. (2020). Consistencia interna y dimensionalidad del cuestionario breve para detectar intimidación escolar. Pensamiento Psicológico, 18(1), 117-125. doi:10.11144/Javerianacali.PPSI18-1.cidc 